\title{
Médicos exiliados en México. Su labor y aportes (1936-1939)
}

\author{
Exiled medics in Mexico. Their work and contributions (1936-1939)
}

José L. Gómez-De Lara*

Facultad de Medicina, Benemérita Universidad Autónoma de Puebla, Puebla, Puebla, México

\begin{abstract}
Resumen
El exilio de republicanos españoles marca uno de los momentos de intercambio cultural más importantes entre México y España. La propuesta de este artículo es analizar la llegada de médicos españoles a tierras mexicanas, las causas que motivaron su salida y sus contribuciones más significativas en el campo de la medicina mexicana. Rafael Méndez, Dionisio Nieto, Isaac Costero, Ramón Álvarez y José Puche fueron cinco de los médicos que más destacaron en el campo de la medicina por sus enseñanzas e investigaciones tras un ciclo de actividad bélica por los campos de Europa. Asimismo, contribuyeron a la formación de nuevas generaciones de investigadores y académicos de la Universidad Nacional de México y de otras instituciones educativas del país.
\end{abstract}

Palabras clave: Exilio español. Medicina. México. Aportes.

\begin{abstract}
The Spanish republican exile marks one of the most important moments of cultural exchange between Mexico and Spain. The proposal of this article is to analyze the arrival of these Spanish in Mexican lands, the causes that motivated their departure, as well as their most significant contributions in the field of Mexican medicine. Rafael Méndez, Dionisio Nieto, Isaac Costero, Ramón Álvarez and José Puche were five of the physicians who most stood out in the field of medicine for their teachings and research after a cycle of war activity in the fields of Europe. Likewise, as they contributed to the training of new generations of researches and academics from the National University of Mexico and others educational institutions on the country.
\end{abstract}

Key words: Spanish exile. Medicine. Mexico. Contributions.

\section{Introducción}

El primero de septiembre de 1939, el general Lázaro Cárdenas del Río rendía «ante Vuestra Soberanía el quinto informe que contiene en extracto las labores desarrolladas por las diversas dependencias del Ejecutivo durante el quinto año del actual gobierno y a exponer a la vez los asuntos principales que en el mismo período se presentaron a su consideración»'. Uno de ellos fue el tema de los refugiados españoles en México. Al respecto, el presidente declaró lo siguiente: «Ante el cumplimiento de deberes universales de hospitalidad y frente a las desgracias colectivas de España, se abrieron las puertas de México a los elementos republicanos que no pueden estar en su patria sin peligro de sus vidas y por considerar,

\section{Correspondencia:}

*José L. Gómez-De Lara

Nogal, 15

Fracc. San Facundo 2

Fecha de recepción: 09-08-2020

C.P. 72764, San Juan Cuautlancingo, Pue., México

E-mail: amoyot।@hotmail.com

Fecha de aceptación: 06-11-2020

DOI: $10.24875 / C I R U .20000880$
Cir Cir. 2021;89(2):278-283
Contents available at PubMed

www.cirugiaycirujanos.com

0009-7411/@ 2020 Academia Mexicana de Cirugía. Publicado por Permanyer. Este es un artículo open access bajo la licencia CC BY-NC-ND (http://creativecommons.org/licenses/by-nc-nd/4.0/). 
además, que se trata de una aportación de fuerza humana y de raza a la nuestra en espíritu y en sangre, que fundida con los aborígenes contribuyó a la formación de nuestra nacionalidad. Espera el Gobierno Federal que pasada la etapa de la agitación preelectoral, que ha esgrimido tal actitud como arma política de oposición, se llegarán a estimar en todo el país los beneficios que recibe México con la aportación de esas energías humanas que vienen a contribuir con su capacidad y esfuerzo al desarrollo y progreso de la Nación²». Con estas palabras, el presidente Lázaro Cárdenas brindaba asilo político a los republicanos españoles que decidieron dejar su país natal. «Esta decisión tuvo como consecuencia el arribo a México de más de 30 mil españoles, que fueron recibidos en el país desde los inicios de la Guerra Civil hasta los años de la posguerra.» Entre los exiliados españoles que arribaron a México durante los años 1937 a 1939 se encontraban profesionistas e intelectuales.

Por iniciativa del historiador y economista Daniel Cosío Villegas (1898-1976), quien se hallaba en Portugal debido a asuntos de negocios, tuvo la gran idea de acoger a los científicos intelectuales españoles para que continuaran con sus actividades científicas mientras finalizaba la Guerra Civil Española, y al mismo tiempo contribuir al desarrollo científico y tecnológico del país que los acogía ¿Quiénes fueron estos científicos españoles? De acuerdo con la tesis de Luis Enrique Vázquez Castillo, Los «intelectuales» españoles transterrados en México, llegaron a la República Mexicana alrededor de 5000 intelectuales $^{3}$, entre los que se encontraban médicos de diversas especialidades.

\section{Llegada de los galenos españoles a tierras mexicanas}

El médico mexicano Salvador Zubirán, durante su viaje de luna de miel, fue comisionado «embajador especialísimo» por el presidente Cárdenas para entrevistarse en París con intelectuales españoles y extender su ayuda a algunos científicos concentrados en los campos franceses. De esta manera, las autoridades mexicanas pretendieron llevar a cabo una generosa operación que fuese provechosa tanto para los vencidos republicanos como para el pueblo mexicano 4 .

A finales de enero de 1939, Daniel Cosío Villegas ya se había anticipado en traer a estos intelectuales a México, para que continuaran con su trabajo y al mismo tiempo contribuyeran al desarrollo científico y tecnológico del país. Los nuevos huéspedes realizaban actividades en ingeniería, arquitectura, química, ciencias exactas, ciencias naturales y medicina, siendo los médicos el grupo más numeroso. Entre los doce primeros invitados que estaban anotados en la lista de Cosío Villegas se encontraban los médicos Gonzalo Rodríguez Lafora (descubridor del cuadro clínico que lleva su nombre: «enfermedad de Lafora»), cuya especialidad fue la Psiquiatría, y el maestro Isaac Costero, especialista en oncología e histología, quien llegó a México a la edad de 36 años. Para el Dr. Francisco Giral, químico farmacéutico y docente español, exiliado en 1939, Costero fue «sin duda, el científico exiliado de más categoría, el que ha producido mayor obra original de calidad, el que ha formado verdadera escuela con mayor número de alumnos distinguidos y cuya obra ha tenido la máxima trascendencia en México»5.

Por supuesto que, con el transcurrir de la guerra y la caída de Madrid en manos de Franco, el listado de invitados se hizo más largo y complejo. A este se unieron «el fisiólogo Jaime Pi-Suñer, el endocrinólogo Rosendo Carrasco Formiguera y el médico Antonio Trías; se unirían después los oftalmólogos Manuel Márquez y Manuel Rivas Cherif, así como el oncólogo Germán García y el psiquiatra Dionisio Nieto» ${ }^{6}$. Muchos de los médicos que llegaron durante el año 1939 no traían consigo los títulos ni los documentos que los acreditaran como médicos profesionistas, por lo que al llegar a México tuvieron el serio problema de certificar su profesión. Fue entonces que el presidente Cárdenas encargó al presidente del recién creado Ateneo Ramón y Cajal, Manuel Márquez Rodríguez, catedrático de Oftalmología de la Facultad de Medicina de la Universidad de Madrid, certificar a los médicos que habían llegado previo examen minucioso en cada caso.

Al pasar el examen, el Ateneo expedía certificados que reemplazaban el título profesional revalidado por autoridades mexicanas, permitiendo así el ejercicio legal de los médicos españoles ${ }^{7}$. Estos médicos, al contar con un título que ratificara su profesión, de inmediato trabajaron en diversas partes de la República Mexicana, como Tamaulipas, Hidalgo, Nuevo León, Veracruz, Yucatán, Jalisco, Puebla y México, y al mismo tiempo dictaron conferencias en la Casa de España, escribieron cuantiosos libros respecto a temas médicos, colaboraron en revistas de medicina como Acta Hidalguense y Archivos médicos mexicanos, y tradujeron al español libros escritos en otros idiomas. En palabras del prominente cardiólogo 
mexicano, el doctor Ignacio Chávez Sánchez (18971979), «los médicos españoles vinieron a México en el momento preciso en que, apenas iniciada la transformación de la medicina dos o tres lustros antes, el ritmo de su avance cobraba impulso»8.

Cuando estos médicos exiliados llegaron a México, la ciencia comenzaba a despuntar y la medicina se encontraba experimentando un proceso de renovación gracias a la creación de nuevas especialidades médicas en el Hospital General, como fue la cardiología a cargo del doctor Ignacio Chávez. Así mismo, se crearon el Instituto de Salubridad y de Enfermedades Tropicales, inaugurado el 18 de marzo de 1939, y el Hospital de Enfermedades de la Nutrición a principios de la década de 1940. Por estas fechas se fundó también el Hospital Infantil Federico Gómez (30 de abril de 1943), cuyo proyecto se había iniciado en el año 1933. Del mismo modo, se iniciaron estudios sobre la función y la patología del sistema nervioso: «una curiosa coincidencia dio lugar a que en los años cuarenta, en México, se desarrollaron con gran ímpetu las ciencias biomédicas relacionadas con el sistema nervioso, lo que hoy conocemos como neurociencias".

Como se puede apreciar, gracias al apoyo en materia educativa por parte de Lázaro Cárdenas y al aporte científico de algunos intelectuales españoles que encontraron refugio laboral en la universidad, «la UNAM entró en un período de fortalecimiento y expansión de institutos de investigación y facultades»", y por supuesto se vivió un momento de esplendor académico. Entre los médicos españoles que destacaron como académicos de la universidad y dieron un fuerte impulso a la medicina mexicana se encuentran cuatro que terminaron sus carreras muy jóvenes y que pasaron a formar parte del personal docente de la UNAM y realizaron labor investigativa en este lugar.

\section{Una vida dedicada a la medicina. El trabajo de los médicos españoles}

El primero de esos cuatro médicos fue el doctor José Puche Álvarez (1895-1979), quien fue presidente del Comité Técnico de Ayuda a los españoles en México, y que al llegar a la Ciudad de México se incorporó inicialmente al Instituto Politécnico Nacional como docente, en los años 1939 a 1946, y tiempo después a la UNAM, de 1945 a 1965. En 1958 ingresó al Departamento de Fisiología de la Facultad de Medicina de la UNAM. En este lugar "formó a un grupo de estudiantes de posgrado a los cuales logró interesar en la fisiología de la regulación de la glucosa y la ingestión de alimentos, temas con los que inició su carrera científica»10. Destacan sus estudios sobre el metabolismo hidrocarbonado en algunos crustáceos del género Procambarus bouvieri cabalbroy y Cambareus moctezumae, demostrando la actividad neuroendocrina de la glándula sinus y su papel en la respuesta glucemiante. Participó en la comisión revisora de los programas de enseñanza de la fisiología y durante el año 1965 organizó un curso para la formación de profesores en fisiología. Murió en 1979 y dejó como legado su extraordinaria biblioteca a la Facultad de Medicina. En el libro España desde México. Vida y testimonio de transterrados, su autora nos presenta una interesante entrevista que realizó al Dr. José Puche antes de fallecer 9 .

El doctor Rafael Méndez Martínez (1906-1991) formó toda una escuela de investigadores de gran renombre en las ciencias biomédicas de México. El Dr. Méndez viajó a los Estados Unidos como exiliado de la Guerra Civil. Lo acogió la Universidad de Harvard, donde fue nombrado instructor e investigador asociado de la Escuela de Medicina. «En 1944, el Dr. Chávez le hace una invitación para ocupar la jefatura del Departamento de Farmacología del Instituto Nacional de Cardiología. Posteriormente, desempeñó sus labores de investigación y docencia como profesor de farmacología en la División de Estudios Superiores de la UNAM. ${ }^{10}$ » A partir de 1975 fue jefe de la División de Investigaciones del Instituto Nacional de Cardiología. Sus aportaciones en la farmacología fueron constantes, concretamente en el campo de los glucósidos digitálicos y los derivados de la veratina, los antiarrítmicos, la circulación coronaria y sus receptores adrenérgicos. Contribuyó a la formación de investigadores mexicanos, como Juan José Mandoki, Pablo Rudomin, Jorge Aceves, Gustavo Pastelín, Carlos Méndez, Davis Erlij, Antonio Morales y Fermín Valenzuela. Falleció en 1991. La autora Ascensión H. de León Portilla le realizó una entrevista que se puede leer en su libro.

El tercer prominente médico fue el doctor Dionisio Nieto Gómez, quien se naturalizó mexicano en 1941. Nació en Madrid, España, el 13 de marzo de 1908, y falleció el 2 de enero de 1985 en la Ciudad de México. Fue un neurólogo y psiquiatra que influyó en el desarrollo de la psiquiatría en México, «paladín del estudio de las bases fisiológicas de las enfermedades mentales al realizar estudios en cerebros provenientes de autopsias del Hospital Psiquiátrico La Castañeda» ${ }^{10}$. En 1964 y 1965 se hizo cargo de la 
jefatura del servicio de psiquiatría e investigaciones cerebrales del Instituto Nacional de Neurología y Neurocirugía, continuando con la formación de generaciones de neurólogos y psiquiatras. Ejerció la cátedra de clínica psiquiátrica y enseñó psicofarmacología de posgrado. Investigó las alteraciones que producía la infección provocada por la tenia llamada solium, contraída por la ingestión de carne contaminada, y en el año 1956 diseñó una reacción de fijación del complemento para el diagnóstico de la cisticercosis del sistema nervioso, conocida como «reacción de Nieto", usada por décadas. A medida que pudo implementar su laboratorio de neurobiología en la UNAM, realizó un estudio cuidadoso sobre la anatomía patológica de la esquizofrenia. Fue el primer psiquiatra en usar clorpromazina en México y antidepresivos tricíclicos para el tratamiento de los trastornos mentales graves. Editó la revista Neurología, Neurocirugía y Psiquiatría en 1959.

Por último, el doctor Isaac Costero Tudanca (19031980), quien desde muy joven se interesó por la histología (estudio de los tejidos), a la cual dedicó la mayor parte de su vida. Al terminar la Guerra Civil tuvo que exiliarse en México, donde fundó y desarrolló la Escuela Mexicana de Patología. Fue un excelente y duro maestro, primero en el Hospital General y después en el Instituto Nacional de Cardiología, donde impartió la cátedra de cardiología por 30 años y desarrolló trabajos sobre histopatología del sistema nervioso. En este mismo lugar investigó sobre la hipertensión arterial, lo que le llevó a proponer una nueva clasificación de los sistemas celulares y la existencia del "cuarto elemento del sistema nervioso» ${ }^{11}$. En 1964, Costero comienza a interesarse por la historia de la anatomía patológica en México y se encuentra con que a él le tocó inaugurar una segunda época dentro de este campo, al incluirla dentro de los planes de estudio de la Escuela de Medicina de la UNAM. "Fue maestro de más de 14 mil alumnos y formó un centenar de patólogos nacionales y extranjeros. Su libro Tratado de anatomía patológica fue un texto obligatorio en muchas facultades de medicina de América Latina. ${ }^{6}$ " En 1979, poco antes de su muerte, fue nombrado Doctor Honoris Causa por la Universidad Nacional Autónoma de México.

Aunque estos cuatro médicos fueron los que más honda huella dejaron en la medicina mexicana, figuraron otros, como «José Ignacio Bolívar y Francisco Bolívar Zapata, que comenzaron a producir sueros contra la tifoidea, el tétano y toxoides antidiftéricos» ${ }^{12}$. Alejandro Otero Fernández fue rector y catedrático de Ginecología en la Universidad de Granada. En México, se hizo cargo de la sala de maternidad del Sanatorio Español e impartió cursos sobre fecundidad y fertilidad en la Sociedad Mexicana de Ginecología y Obstetricia. El médico especialista en ginecología, José Torre Blanco, fue uno de los fundadores de esta sociedad y «se encargó de la dirección de la División de Ginecoobstetricia del Hospital 20 de Noviembre, donde desarrolló parte de sus investigaciones sobre el embarazo extrauterino y sobre la relación entre el riñón y el embarazo»8.

El académico Germán García García, médico español exiliado en 1939, destacó en el campo de la oncología. Al ingresar en el Hospital Morelos fundó, con multitud de dificultades, el Servicio de Detección de Cáncer Ginecológico y también el primer Laboratorio de Citopatología, apoyado entonces por el filántropo español Santiago Galas. Fue uno de los pioneros en el uso de la técnica de Papanicolaou para la detección del cáncer cervicouterino. Inauguró la cátedra de Oncología en la Escuela Superior de Medicina del Instituto Politecnico Nacional (IPN) e inició el servicio de cancerología en este mismo lugar.

En oftalmología figuró Manuel Rivas Cherif, quien fue jefe del servicio del Hospital de la Asociación para Evitar la Ceguera. Realizó investigaciones sobre la fotografía de las membranas profundas del ojo, fotoftalmología, agudeza visual, el insomnio de origen ocular y la anestesia en la operación de cataratas.

El uso de un tinte especial para ver cómo fluye la sangre a través del cerebro (arteriografía cerebral) «progresó con las aportaciones de Jesús Sánchez Pérez. La especialidad médica encargada del estudio de la función de la piel o dermatología, recibió en Julio Bejarano Lozano, maestro e investigador de calidad extraordinaria, un impulso importante al ser elegido presidente de la Sociedad Mexicana de Dermatología y haber publicado más de cien trabajos sobre el tema de la lepra. Los doctores Victoriano M. de Acosta y Pelayo Vilar Canales influyeron en la otorrinolaringología» ${ }^{12}$.

Fueron varios los especialistas en psiquiatría que llegaron a México. En este rubro destacaron Wenceslao López Albo, quien desempeñó como profesor de Psiquiatría en la Universidad de Nuevo León y fue jefe de Neuropsiquiatría en el Hospital Español, en la ciudad de México. Federico Pascual del Roncal se distinguió como el impulsor de una especialidad desconocida 
en México hasta antes de su arribo: la neuropsiquiatría infantil; fue jefe del servicio psiquiátrico en el Instituto Médico Pedagógico y subdirector de la Clínica Neuropsiquiátrica Falcón.

En la especialidad médica que estudia al niño y sus enfermedades, la pediatría, figuraron los especialistas Antonio Encinas Rodríguez, «quien fue jefe de pediatría del Hospital Español e investigador en materia de factor Rh y nutrición de lactantes, y Antonio Palacios Martos y Jesús de Miguel Sancho, quienes promovieron la rehidratación de infantes a través de transfusiones de plasma y sangre ${ }^{12}$.

En el campo de la historia y de la ciencia destacaron tres intelectuales españoles que contribuyeron al desarrollo de la ciencia en México con sus aportaciones. El primero de ellos es el médico Francisco Guerra Pérez-Carral (1916-2011), quien participó en la Guerra Civil y se graduó en Farmacología en la Universidad de Yale. En México, demostró el efecto de los glicósidos cardiotónicos sobre la miosina del miocardio de buey, aumentando su fuerza contráctil al estimular la liberación de fósforo. Fue profesor de Farmacología en la Facultad de Medicina de la UNAM, y escribió y publicó una vasta literatura sobre temas relacionados con la historia de la Medicina, principalmente en México. Se le recuerda por los libros Bibliografía de la materia médica mexicana, publicado en 1950; Bibliografía de la historia de la medicina mexicana, publicado en 1949; Iconografía médica mexicana: Catálogo gráfico descriptivo de los impresos médicos mexicanos de 1552 a 1833, ordenados cronológicamente, publicado en España en 1955; y Las heridas de guerra. Contribución de los cirujanos españoles en la evolución de su tratamiento, publicado en 1981, entre otros títulos. Con más de 50 obras de su autoría, entre libros y artículos, el doctor Francisco Guerra impulsó el estudio de la historia de la medicina en México y en el mundo. Falleció en Madrid a los 95 años.

El Dr. Modesto Bargalló Ardévol (1894-1981) fue un químico español que trabajó 40 años en México. Catedrático del Instituto Politécnico Nacional, se dedicó a la historia de las industrias extractivas en México, particularmente del periodo colonial. Así mismo, tuvo una actividad reconocida como historiador de la química, sobresaliendo en estudios sobre la minería y la metalurgia prehispánica, colonial y de la independencia temprana, así como del conocimiento que los pueblos originarios de esta región tenían sobre dichas materias, las técnicas de beneficio de los metales, ordenanzas reguladoras dictadas por los gobiernos y otros aspectos afines. A la edad de 87 años, falleció en la Ciudad de México.

El doctor Germán Somolinos D'Ardois (1911-1973) desarrolló a lo largo de su trayectoria profesional el rescate, el registro, el estudio y la compilación de diversas fuentes para el estudio de la Medicina en México. Nació en Madrid y murió en la Ciudad de México. Cursó estudios de Medicina en la Universidad Central de Madrid. Por la situación que estaba pasando España, pasó a México en 1938 y rápidamente se integró a las tareas de la Facultad de Medicina de la UNAM, del Instituto Nacional de Cardiología y del Instituto Politécnico Nacional, y por supuesto dirigió su trabajo al análisis de la historia científica de la Medicina en México, tanto de los métodos y los aspectos de la salud pública como de las biografías y los legados de importantes médicos mexicanos y extranjeros. Entre sus aportaciones relevantes se encuentra la instalación de un laboratorio de análisis clínicos en su casa, y así realizó por primera vez en México estudios sobre $\mathrm{Rh}$, grupos sanguíneos y eritroblastosis. Igualmente, introdujo la técnica para realizar la electroforesis para el estudio de las lipoproteínas. En el año 1956, en compañía de otros historiadores de la Medicina, fundaron la actual Sociedad Mexicana de Historia y Filosofía de la Medicina, de la cual llegó a ser presidente. Falleció en 1973 a los 62 años de edad, y quedó a cargo del laboratorio su hijo, el doctor Juan Somolinos Palencia, importante historiador y difusor de la historia de la Medicina en México, quien destacó por la cantidad de obra escrita (300 artículos en revistas como La Gaceta Médica de México y Revista Médica del Instituto Mexicano del Seguro Social, entre otras) y por su dedicación a la Sociedad Mexicana de Historia y Filosofía de la Medicina, en la cual fungió como presidente de 1980 a 1982.

Con respecto a los médicos ortopedistas españoles que se establecieron en México, me encontré únicamente con la figura de José Montalvo Villar (19071973). Nacido en Linares, en la provincia de Jaén, fue un mecánico ortopédico que sufrió durante la Guerra Civil la amputación de tres dedos de la mano izquierda. Llegó exiliado a la República Dominicana con su esposa y tres hijos, y pudo obtener en el consulado de México, en ciudad Trujillo (Santo Domingo), la visa como asilado político el 14 de diciembre de 1943 a instancias de la Federación de Organizaciones de Ayuda a Refugiados Europeos, e ingresar en México pocas semanas después. Proyectó instalar un taller ortopédico en la Ciudad de México con la ayuda de la Junta de Ayuda de Republicanos Españoles. 
Falleció de trauma en México y fue enterrado en el Panteón Español.

\section{Conclusión}

En el presente texto he presentado algunos de los médicos que destacaron en sus respectivos campos, pero hubo otros que no alcancé a mencionar, y claro, merecen todo nuestro reconocimiento por su aporte a la medicina mexicana. En este grupo se encuentran cuatro mujeres: María del Coro Clavero Maestre (quien vino a México con sus dos hijos, Nora y Luis Julián Berdejo Clavero), Úrsula Mayer Van Beijeren, Cecilia Sanz Sanz y Eladia Faraudo Puigdollers. De las tres primeras, no encontré información sobre su vida o actividad profesional en México. De Eladia Faraudo Puigdollers se sabe que trabajó en la Generalidad de Cataluña como directora de Asistencia Social. A causa de la Guerra Civil, llegó al puerto de Veracruz en 1939 y de ahí se trasladó a Monterrey, donde se incorporó a la Universidad de Nuevo León y participó en la fundación de la Escuela de Trabajo Social de Nuevo León y del Servicio de Trabajo Social del Hospital Universitario de Monterrey. Murió en el año 1979 en México.

Lo que hay que recalcar es que ellas, como el resto de los demás médicos, encontraron suelo fértil para su trabajo y se agruparon en diversas instituciones donde pudieron realizar libremente su trabajo. Todo fue gracias al apoyo a la educación superior y a la investigación científica por parte del general Lázaro Cárdenas, en cuya cabeza tenía la firme idea de que la educación tenía que ser reflejo y factor de transformación de la sociedad.

\section{Agradecimientos}

El autor agradece al médico potosino Carlos Agustín Rodríguez-Paz por sus consejos y sugerencias para el desarrollo de este trabajo, así como por proporcionarle datos e información relevante con respecto al tema.

\section{Financiamiento}

La presente investigación no ha recibido ningún tipo de financiación ni ayuda económica.

\section{Conflicto de intereses}

El autor declara no tener conflictos de intereses.

\section{Bibliografía}

1. Cárdenas del Río L. V Informe de Gobierno del Presidente Constitucional de los Estados Unidos. $1^{\circ}$ de septiembre de 1939. p. 179. (Consultado el 5 de mayo de 2020.) Disponible en: http://www.diputados.gob. $\mathrm{mx} /$ cedia/sia/re/RE-ISS-09-06-08.pdf/.

2. Centro Republicano Español de México. México y la República Española. Antología de documentos. 1931-1977. México; 1978.

3. Vázquez Castillo LE. La postura de México ante la Guerra Civil. Los "intelectuales" españoles transterrados en México. Benemérita Universidad Autónoma de Puebla, Facultad de Filosofía y Letras, Tesis para obtener el grado de Licenciado en Historia. México; 2012. p. 21-48.

4. Puerto J. Los Giral. La Casa de España y el exilio mexicano. Los refugiados españoles y la cultura mexicana: actas de las jornadas celebradas en España y México para conmemorar el septuagésimo aniversario de la Casa de España en México (1938-2008). Colegio de México. México; 2010.

5. Martínez Palomo, A. Cárdenas, la ciencia y el exilio español en México. Letras libres; 2006;61:31.

6. Sánchez-Albornoz N. El destierro español en América. Un trasvase cultural. Sociedad Estatal Quinto Centenario/Instituto de Cooperación Iberoamericana. México; 1991.

7. Sánchez Díaz G, García de León P. Los científicos del exilio español en México. Instituto de Investigaciones Históricas de la Universidad Michoacana de San Nicolás de Hidalgo/Sociedad Mexicana de Historia de la Ciencia y de la Tecnología. México; 2001.

8. Somolinos D'Ardois G. 25 años de medicina española en México. Trabajo de la Sección de Historia de la Medicina leído en la sesión del 26 de mayo de 1965 en la Academia Nacional de Medicina de México, y publicado como folleto por el Ateneo Español de México. México; 1966.

9. H. de León Portilla A. España desde México. Vida y testimonio de transterrados. Universidad Nacional Autónoma de México. México; 1978.

10. Fernández Guardiola A. Las neurociencias en el exilio español en México. La Ciencia para todos, Núm. 153. Fondo de Cultura Económica/ Secretaría de Educación Pública/CONACYT. México; 1997.

11. Isabel Souza M, Pérez Tamayo R, Ávila D. Isaac Costero. Vivir para la ciencia. Academia Nacional de Medicina/CONACYT/Intersistemas. Colección Aniversario 150 años. México; 2014.

12. Serrano Migallón $F$. La inteligencia peregrina: legado de los intelectuales del exilio republicano español en México. El Colegio de México. México; 2009. 